\title{
Erratum: Modeling Gel Swelling Equilibrium in the Mean Field: From Explicit to Poisson-Boltzmann Models [Phys. Rev. Lett. 122, 208002 (2019)]
}

\author{
Jonas Landsgesell@, David Sean, Patrick Kreissl, Kai Szuttor, and Christian Holm®
}

(Received 15 January 2020; accepted 24 February 2020; published 20 March 2020)

DOI: 10.1103/PhysRevLett.124.119901

In our recent publication [1], we revisited our new gel models which we had discussed in this Letter. Closer inspection of the previously used periodic gel simulations [2,3] revealed that they used different FENE bond parameters than our single-chain cell-gel simulations, i.e., $k_{\mathrm{FENE}}=10 k_{B} T / \sigma^{2}$ and $R_{\max }=1.5 \sigma$ were used, while our single-chain cell-gel model (CGM) used the original Kremer-Grest parameters [4] $k_{\mathrm{FENE}}=30 k_{B} T / \sigma^{2}$ and $R_{\max }=1.5 \sigma$. Therefore, the data comparison was based on unequal footing.

Additionally, we found an implementation error in the grand-canonical coupling used in the previous periodic gel simulations by Košovan and Richter [2,3]. We therefore regenerated the periodic gel model data with the Kremer-Grest bond parameters and a corrected grand-canonical simulation scheme [1]. Compared to the flawed data in this Letter, the corrected periodic gel data show improved agreement with the single-chain CGM, see modified Figs. 2 and 3 which replace Figs. 2 and 3 in the Letter. The main conclusions of this Letter are not altered, in fact the usefulness of both models is even more evident.
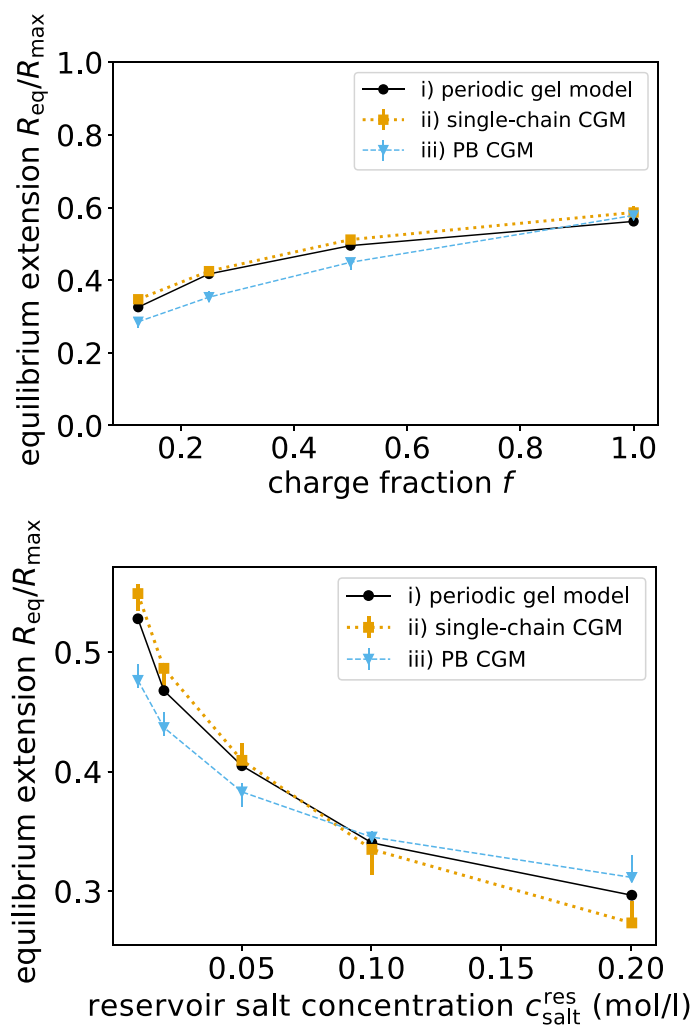

FIG. 2. Comparison between the three models. The equilibrium swelling length $R_{\text {eq }}$ as a function of (a) the charge fraction $f$ along the gel polymer backbone for $c_{\text {salt }}^{b}=0.01 \mathrm{~mol} \mathrm{~L}^{-1}$ and $N \approx 80$; (b) the salt bath concentration $c_{\text {salt }}^{b}$ for $f=0.5$ and $N=64$. As can be seen in Fig. 3 the quality of the predictions of the single-chain model and the PB model for other parameter combinations are also very good. 


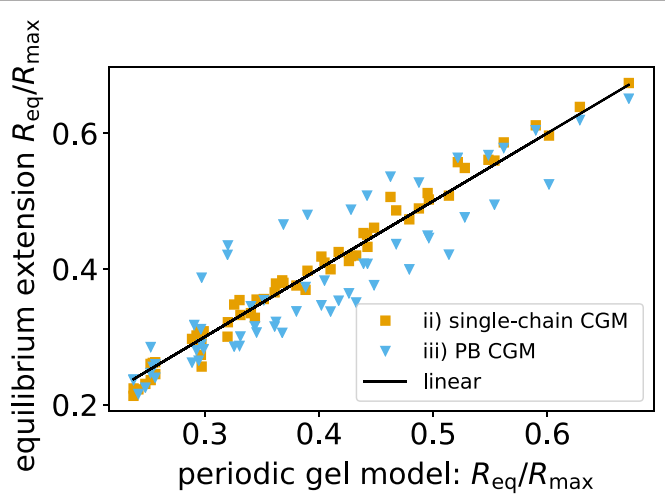

FIG. 3. The swelling equilibria of the single-chain cell model and the PB model compared to the periodic gel simulations. The results are compared for a wide exploration of in total 60 parameter combinations with $N \approx 40,64,80, f \in\{0.125,0.25,0.5,1\}$ and $c_{\text {salt }}^{b} \in\{0.01,0.02,0.05,0.1,0.2\} \mathrm{molL}^{-1}$. The linear function has the form $y(x)=x$.

C. H. acknowledges financial contributions from the Deutsche Forschungsgemeinschaft (DFG, German Research Foundation) under Germany's Excellence Strategy - EXC 2075 - 390740016, and 423435431 as part of FOR2811, and from Grants No. HO 1108/26-1 and No. AR 593/7-1.

[1] J. Landsgesell and C. Holm, Cell model approaches for predicting the swelling and mechanical properties of polyelectrolyte gels, Macromolecules 52, 9341 (2019).

[2] P. Košovan, T. Richter, and C. Holm, Modeling of polyelectrolyte gels in equilibrium with salt solutions, Macromolecules 48, 7698 (2015).

[3] T. Richter, Coarse grained hydrogels, Ph.D. thesis, University of Stuttgart, 2017, http://dx.doi.org/10.18419/opus-9203.

[4] G. S. Grest and K. Kremer, Molecular dynamics simulation for polymers in the presence of a heat bath, Phys. Rev. A 33, 3628 (1986). 